\title{
CHARACTERS AND ORTHOGONALITY IN FROBENIUS ALGEBRAS
}

\author{
T. V. Fossum
}

\begin{abstract}
Matrix-theoretical proofs of orthogonality relations for the coefficients of representations of Frobenius algebras have been extensively developed in the literature. This paper has grown out of a desire to prove some of these orthogonality relations in a matrix-free, module-theoretic manner. Most of the attention is focused on characters, even though some of the results diverge from this restriction.
\end{abstract}

Sections 1 and 2 set the stage for the development of the principal theorems in $\S 3$. There we derive the orthogonality relations and demonstrate a relation between characters and certain homogeneous modules over Frobenius algebras. In $\S 4$ we apply these results to obtain some information about the character of the left regular module.

All rings are assumed to have an identity, and all modules are assumed to be unital. The Jacobson radical of a ring $A$ will be written $J(A)$, or simply $J$.

Let $A$ be a ring, $M$ a simple left $A$-module, and let $A_{M}=\{a \epsilon$ $A: a M=0\}$. Then $A_{M}$ is a two-sided ideal of $A$, the annihilator of $M$. If $L$ is a left $A$-module, $\operatorname{Soc}_{M}(L)$ will denote the $M$-socle of $L$, i.e., the sum of all submodules of $L$ isomorphic to $M$. We say $L$ is $M$-homogeneous if $\operatorname{Soc}_{M}(L)=L$. In particular if $A$ is left artinian, then $A_{M}$ is a maximal two-sided ideal of $A$, and $L$ is $M$-homogeneous if and only if $A_{M} L=0$. We let $S_{M}$ denote the $M$-socle $\operatorname{Soc}_{M}(A)$ of $A$. A block of $A$ is an indecomposable ring direct summand of $A$ [2, $\S 55]$. We say $M$ belongs to the block $B$ of $A$ if $M$ is a composition factor of $B$ regarded as a left $A$-module.

Let $K$ be a field. If $V$ is a vector space over $K$, let $(V: K)$ denote the $K$-dimension of $V$. Now assume $A$ is a finite dimensional $K$-algebra. Then $A^{*}=\operatorname{Hom}_{K}(A, K)$ is an $(A, A)$-bimodule, where for $a \in A$ and $\lambda \in A^{*}$ we define

$$
\left\{\begin{array}{l}
(a \lambda)(x)=\lambda(x a) \\
(\lambda a)(x)=\lambda(a x) .
\end{array}\right.
$$

If $\lambda \in A^{*}$, we say $\lambda$ is a class function if $\lambda(a b)=\lambda(b a)$ for all $a, b \in A$. Let $\operatorname{cf}(A)$ denote the set of class functions in $A^{*}$. Observe that $\lambda \in$ $\operatorname{cf}(A)$ if and only if $a \lambda=\lambda a$ for all $a \in A$. We say $\chi \in A^{*}$ is an $A$ character if $\chi$ is the character of a (finite dimensional) left $A$-module. Clearly all $A$-characters belong to $\mathrm{cf}(A)$. The $A$-character $\chi$ is said to be irreducible if it is the character of a simple left $A$-module. 
Definition. Let $A$ be a finite-dimensional $K$-algebra, $K$ a field, and let $M$ be a simple left $A$-module. We say an element $\lambda \in A^{*}$ belongs to $M$ if $\lambda\left(A_{M}\right)=0$. One checks that if $\lambda$ belongs to $M$, then $a \lambda$ and $\lambda a$ belong to $M$ for any $a \in A$. If $\chi$ is the character of $M$, then $\chi$ clearly belongs to $M$.

All other notation may be found in [2] or [3].

1. Socles of $Q F$. rings. A ring $A$ is said to be a $Q F$ (quasiFrobenius) ring if $A$ is (left and right) artinian and ${ }_{A} A$ is injective $[2,(58.6)]$.

Lemma 1.1. Let $A$ be a $Q F$ ring, $M$ a simple left $A$-module, and $S_{M}=\operatorname{Soc}_{M}(A)$. Then $S_{M}$ is a nonzero simple two-sided ideal of $A$.

Proof. By [2, (58.13)], $M$ is isomorphic to a minimal left ideal $I$ in $A$, so $S_{M} \neq 0$. Moreover $S_{M}=\sum\left\{f(I): f \in \operatorname{Hom}_{A}(I, A)\right\}$ since $I \cong M$. By assumption ${ }_{A} A$ is injective and from the "injective test lemma" [2, (57.14)] it follows that each $f \in \operatorname{Hom}_{A}(I, A)$ is right multiplication by some $a \in A$; that is, $f(I)=I a \leqq I A$. Therefore $S_{M} \leqq I A$. But plainly $I A \subseteq S_{M}$, and the lemma follows.

Lemma 1.2. Let $A$ be a $Q F$ ring, $M$ a simple left $A$-module, and $S_{M}=\operatorname{Soc}_{M}(A)$. Then the following are equivalent.

(a) $S_{M}$ is a simple block of $A$ to which $M$ belongs

(b) $S_{M}^{2} \neq 0$

(c) $M$ is projective.

Proof. That (a) implies (b) is obvious. For (b) implies (c), suppose ' $M$ is not projective. By [2, (58.12)] $M$ is isomorphic to the "bottom" constituent $I$ of a principal indecomposable left ideal $U$, and as such, $I \subseteq J U$ where $J$ is the raidcal of $A$. Hence $I \subseteq J$, and since $I \subseteq S_{M}$, $S_{M} \subseteq J$. But $S_{M}$ is $M$-homogeneous and $J \subseteq A_{M}$ so therefore

$$
S_{M}^{2} \subseteq A_{M} S_{M}=0 .
$$

To show that (c) implies (a), notice that if $M$ is projective, then $M$ is injective [2, (58.14)] and therefore $M$ is a direct summand of every module with $M$ as a composition factor. Therefore every principal indecomposable module linked to $M$ is isomorphic to $M$, so $S_{M}$ is a block of $A[2, \S 55]$. From (1.1) it follows that $S_{M}$ is simple, completing the proof.

2. Frobenius algebras and class functions. Let $A$ be a finitedimensional $K$-algebra, $K$ a field. We say that $A$ is a Frobenius algebra if $A \cong A^{*}=\operatorname{Hom}_{K}(A, K)$ as left $A$-modules. To make this 
connection more explicit, we say that the pair $(A, \varphi)$ is a Frobenius algebra if $\varphi: A \rightarrow A^{*}$ is a left $A$-isomorphism (see [4] and [2, (61.1)].) By [2, (61.1), (61.2)] a Frobenius algebra is necessarily a $Q F$ ring; hence the results of $\S 1$ apply.

Let $(A, \varphi)$ be a Frobenius algebra. If $\left(a_{i}\right),\left(b_{i}\right)$ is an ordered pair of bases for $A$ such that $\varphi\left(b_{i}\right)\left(a_{j}\right)=\delta_{i j}$ for all $i, j$, we say the bases $\left(a_{i}\right),\left(b_{i}\right)$ are $\varphi$-dual. If $\left(a_{i}\right)$ is any basis for $(A, \varphi)$, there exists a second basis $\left(b_{i}\right)$ such that $\left(a_{i}\right),\left(b_{i}\right)$ are $\varphi$-dual. (In most cases we are given an explicit isomorphism $\varphi$ and certain $\varphi$-dual bases, although for an arbitrary Frobenius algebra $A$ there may be many different choices for $\varphi$ or $\varphi$-dual bases.)

LEMMA 2.1. Let $\left(a_{i}\right),\left(b_{i}\right)$ be $\varphi$-dual bases for the Frobenius algebra $(A, \varphi)$, and assume $\lambda \in A^{*}$. Then $\varphi^{-1}(\lambda)=\sum_{2} \lambda\left(a_{i}\right) b_{i}$.

Proof. The proof is left to the reader.

Lemma 2.2. Let $(A, \varphi)$ be a Frobenius algebra. Then the mapping $\tau=\tau_{\varphi}: A \rightarrow A$ given by $\tau(a)=\varphi^{-1}(\varphi(1) a)$ is a K-algebra automorphism of $A$.

Proof. Observe that $\varphi(\tau(a))=\varphi(1) a$ for each $a \in A$. Because $\varphi$ is a left $A$-homomorphism and $A^{*}$ is an $(A, A)$-bimodule, it follows easily that

$$
\varphi(x \tau(a))=\varphi(x) a, \quad(x, a \in A) .
$$

If $x=\varphi^{-1}(\lambda)$ for $\lambda \in A^{*}$, taking $\varphi^{-1}$ on both sides of (2.3) gives

$$
\varphi^{-1}(\lambda) \tau(\alpha)=\phi^{-1}(\lambda \alpha), \quad(a \in A) .
$$

Now for $a, b \in A, \varphi(\tau(a b))=\varphi(1) a b=\varphi(\tau(a)) b=\varphi(\tau(a) \tau(b))$ by (2.3), and therefore since $\varphi$ is one-to-one, $\tau(a b)=\tau(a) \tau(b)$. If $\tau(a)=0$, then $0=\varphi(\tau(a))=\varphi(1) a$, so $\varphi(b) a=b(\varphi(1) a)=0$ for all $b \in A$. But then $0=(\varphi(b) a)(1)=\varphi(b)(a)$ for all $b \in A$, and it follows that $a=0$ since $\varphi(A)=A^{*}$. Therefore $\tau$ is one-to-one. Verification of the remaining properties of $\tau$ is left to the reader.

The reader may observe that $\tau=\tau_{\varphi}$ is the inverse of "Nakayama's automorphism" [5, Th. 1].

Definition. Let $(A, \varphi)$ be a Frobenius algebra with automorphism $\tau=\tau_{\varphi}$ as in (2.2). Define $Z_{\tau}(A)=\{a \in A: b a=a \tau(b)$ for all $b \in A\}$,

Lemma 2.5, Let $(A, \varphi)$ be a Frobenius algebra with automorphism $\tau=\tau_{\varphi}$ as in (2.2). Then $\varphi^{-1}(\operatorname{cf}(A))=Z_{\tau}(A)$. 
Proof. If $a \in Z_{\tau}(A)$, then $b a=a \tau(b)$ for all $b \in A$. Then by (2.3), $b \varphi(a)=\varphi(b a)=\varphi(a \tau(b))=\varphi(a) b$ for all $b \in B$, so $\varphi(a) \in \operatorname{cf}(A)$. The converse is similar.

Corollary 2.6. Let $(A, \varphi), \tau$ be as in (2.5). If $\lambda \in \operatorname{cf}(A)$ and $\lambda^{*}=\varphi^{-1}(\lambda)$, then $A \lambda^{*}=\lambda^{*} A$.

Proof. By (2.5), $\lambda^{*} \in Z_{\tau}(A)$. Since $\tau$ is an automorphism of $A$, $A \lambda^{*}=\lambda^{*} \tau(A)=\lambda^{*} A$.

3. Orthogonality relations, socles and characters. Throughout this section let $(A, \varphi)$ be a Frobenius algebra. Recall that if $M$ is a simple left $A$-module, then $A_{M}=\{a \in A: a M=0\}$, and for $\lambda \in A^{*}, \lambda$ belongs to $M$ if $\lambda\left(A_{M}\right)=0$.

Lemma 3.1. Let $M$ be a simple left A-module, and assume $\lambda$ belongs to $M, \lambda \in A^{*}$. Set $\lambda^{*}=\varphi^{-1}(\lambda)$. Then $A_{M} \lambda^{*}=0$. In particular if $N$ is any left $A$-module, then $A \lambda^{*} N$ is $M$-homogeneous.

Proof. If $b \in A_{M}$, then $b \lambda=0$ because $(b \lambda)(A)=\lambda(A b) \leqq \lambda\left(A_{M}\right)=0$. Therefore $b \lambda^{*}=\varphi^{-1}(b \lambda)=0, b \in A_{M}$, and so $A_{M} \lambda^{*}=0$. For $N$ a left $A$-module, $A_{M}(A \lambda * N)=A_{M} \lambda^{*} N=0$, so $A \lambda * N$ is $M$-homogeneous.

THEOREM 3.2. (Orthogonality relations). Let $M$ and $N$ be simple left $A$-modules, and assume $\lambda, \mu$ belong to $M, N$, respectively, where $\lambda, \mu \in A^{*}$. Set $\lambda^{*}=\varphi^{-1}(\lambda)$. If $\lambda^{*} N \neq 0$, then $M \cong N$. In particular if $\mu\left(\lambda^{*}\right) \neq 0$, then $M \cong N$.

Proof. Suppose $\lambda^{*} N \neq 0$. Since $N$ is simple, $A \lambda * N=N$. By (3.1), $N$ must then be $M$-homogeneous, i.e., $M \cong N$. In particular if $\mu\left(\lambda^{*}\right) \neq 0$, then plainly $\lambda^{*} N \neq 0$, so by above $M \cong N$.

CoROllary 3.3. Let $M, N, \lambda, \mu$ be as in (3.2), and set

$$
\lambda^{*}=\varphi^{-1}(\lambda), \mu^{*}=\varphi^{-1}(\mu) \text {. }
$$

If $\lambda^{*} \mu^{*} \neq 0$, then $M \simeq N$.

Proof. Assume $\lambda^{*} \mu^{*} \neq 0$. Then $\lambda^{*} \mu \neq 0$, and therefore for some $a \in A, 0 \neq\left(\lambda^{*} \mu\right)(\alpha)=\mu\left(a \lambda^{*}\right)=(\mu a)\left(\lambda^{*}\right)$. But $\mu a$ belongs to $N$, so by (3.2), $M \cong N$.

COROLLARY 3.4. (Orthogonality relations for characters). Let $M, N$ be simple left $A$-modules with characters $\chi$ and $\zeta$, respectively. Set $\chi^{*}=\varphi^{-1}(\chi)$. If $\zeta\left(\chi^{*}\right) \neq 0$, then $M \cong N$ and $\chi=\zeta$. In particular if $\left(a_{i}\right),\left(b_{i}\right)$ are $\varphi$-dual bases for $A$, and if $\sum_{i} \chi\left(a_{i}\right) \zeta\left(b_{i}\right) \neq 0$, then $M \cong N$ and $\chi=\zeta$. 
Proof. The first part follows directly from (3.2) since the character of a simple module belongs to that module. Moreover modules uniquely determine their characters. For the second part we need only apply this and (2.1).

THEOREM 3.5. Let $\chi$ be the character of the simple left A-module $M$, and assume $\chi \neq 0$. Set $\chi^{*}=\varphi^{-1}(\chi)$. Then $A \chi^{*}=\operatorname{Soc}_{M}(A)=S_{M}$. In particular either $\left(A \chi^{*}\right)^{2}=0$, or $A \chi^{*}$ is a simple block of $A$.

Proof. Since $\chi \in \operatorname{cf}(A),(2.6)$ implies that $A \chi^{*}=\chi^{*} A=A \chi^{*} A$. By (3.1), $A \chi^{*}$ is $M$-homogeneous, so $0 \neq A \chi^{*} \subseteq S_{M}$. Since $S_{M}$ is a simple two-sided ideal (1.1), $A \chi^{*}=S_{M}$. The second assertion follows from this and (1.2).

Corollary 3.6. Let $\chi^{*}$ be as in (3.5). If $\left(\chi^{*}\right)^{2}=0$, then $S_{M}^{2}=$ 0. If $\left(\chi^{*}\right)^{2} \neq 0$, then $\chi^{*}$ is a unit in the simple block $A \chi^{*}=S_{M}$.

Proof. By (3.5), $A \chi^{*}=S_{M}$. If $\left(\chi^{*}\right)^{2}=0$, then by (2.6),

$$
S_{M}^{2}=A \chi^{*} A \chi^{*}=A\left(\chi^{*}\right)^{2}=0 .
$$

On the other hand if $\left(\chi^{*}\right)^{2} \neq 0$, then $S_{M}^{2} \neq 0$, so by (1.2) $S_{M}=A \chi^{*}$ is a simple block of $A$ in which $\chi^{*}$ is clearly a unit.

Corollary 3.7. Let $\chi, \chi^{*}$ be as in (3.5). If $\chi\left(\chi^{*}\right) \neq 0$, then $\chi^{*}$ is a unit in the block $A \chi^{*}=S_{M}$.

Proof. If $\chi\left(\chi^{*}\right) \neq 0$, then $\chi^{*} \chi \neq 0$ (in $A^{*}$ ), so by applying $\varphi^{-1}$ we have $\left(\chi^{*}\right)^{2}=\chi^{*} \varphi^{-1}(\chi)=\varphi^{-1}\left(\chi^{*} \chi\right) \neq 0$. By (3.6), $\chi^{*}$ is a unit in $S_{M}$.

REMARK. The converse to (3.7) is false. For example let $F$ be a field of characteristic $p \neq 0$, and let $F_{p}$ be the complete ring of $p$-by- $p$ matrices over $F$. Then $F_{p}$ is a Frobenius algebra with isomorphism $\varphi$ given by $\varphi(X)(Y)=\operatorname{trace}(Y X), X, Y \in F_{p}$. One checks that if $\chi$ is the character of the unique simple left $F_{p}$-module $M$, then $\chi^{*}=\varphi^{-1}(\chi)=I_{p}$ where $I_{p}$ is the $p$-by- $p$ identity matrix. Then $\chi\left(\chi^{*}\right)=p=0$ since $F$ has characteristic $p$, but $\chi^{*}$ is clearly a unit in $F_{p}$.

The procedure for finding the socle of the left regular module ${ }_{A} A$ of a Frobenius algebra (3.5) may also be applied to arbitrary left $A$-modules and in particular to principal indecomposable modules [2, (54.3)]. 
Proposition 3.8. Let $M, \chi^{*}$ be as in (3.5), and assume $N$ is any left A-module. If $\left(\chi^{*}\right)^{2} \neq 0$, then $\chi^{*} N=\operatorname{Soc}_{M}(N)$. If $\left(\chi^{*}\right)^{2}=0$, then $\chi^{*} N \cong \operatorname{Soc}_{M}(J N)$ where $J$ is the radical of $A$.

Proof. Assume $\left(\chi^{*}\right)^{2} \neq 0$. Then $S_{M}=\chi^{*} A$ is the simple block of $A$ to which $M$ belongs (3.6), and $S_{M}+A_{M}=A$. If $L$ is any $M$ homogeneous left $A$-module, then $L=A L=S_{M} L=\chi^{*} L$. By (3.1), $A \chi^{*} N=\chi^{*} N$ is $M$-homogeneous, so $\chi^{*} N \cong \operatorname{Soc}_{M}(N)$. But by above $\operatorname{Soc}_{M}(N)=\chi^{*} \operatorname{Soc}_{M}(N) \subseteq \chi^{*} N$. Therefore $\chi^{*} N=\operatorname{Soc}_{M}(N)$. Now if $\left(\chi^{*}\right)^{2}=0$, then $\chi^{*} \in J=J(A)$ (since $\chi^{*} \in S_{M}$ and $S_{M}^{2}=0$ by (3.6)) so that $\chi^{*} N \leqq J N$. But $\chi^{*} N$ is $M$-homogeneous by (3.1), and therefore $\chi^{*} N \cong \operatorname{Soc}_{M}(J N)$.

EXAMPLE. Equality does not necessarily occur in the relation $\chi^{*} N \subseteq \operatorname{Soc}_{M}(J N)$ when $\left(\chi^{*}\right)^{2}=0$. For example let $G$ be an abelian group of order a prime $p>2$, and let $F$ be a field of characteristic $p$. Then the group-algebra $F G$ is a commutative local ring which is clearly a Frobenius algebra [2, Remark 2, p. 440] with isomorphism $\varphi$, say. Moreover if $J$ denotes the radical of $F G$, then $J^{p}=0$ and $J^{p-1}=\operatorname{Soc}(F G)$.

Let $M$ be a simple left $F G$-module with character $\chi$. Then all simple left $F G$-modules are isomorphic to $M$, and in particular

$$
J^{p-1}=\operatorname{Soc}_{M}(F G) \cong M .
$$

If $\chi^{*}=\varphi^{-1}(\chi)$, then $\chi^{*} \in \operatorname{Soc}_{M}(F G)=J^{p-1}$, and $\left(\chi^{*}\right)^{2}=0$. Let $N=J$, viewed as a left $F G$-module. Then $\chi^{*} N \subseteq J^{p-1} J=J^{p}=0$, so $\chi^{*} N=$ 0 . But $\operatorname{Soc}_{M}(J N)=\operatorname{Soc}_{M}\left(J^{2}\right)=J^{p-1} \neq 0$. Hence $\chi^{*} N \neq \operatorname{Soc}_{M}(J N)$.

THEOREM 3.9. Let $f$ be a primitive idempotent in $A$, and let $M$ be a simple left $A$-module with character $\chi \neq 0$. Set $\chi^{*}=\varphi^{-1}(\chi)$. Then $\chi^{*} f \neq 0$ if and only if $\chi^{*} A f=\operatorname{Soc}(A f) \cong M$.

Proof. By [2, (58.12)], Soc $(A f)$ is simple. If $\chi^{*} f \neq 0$, then $0 \neq A \chi^{*} A f=\chi^{*} A f \subseteq \operatorname{Soc}_{M}(A f) \subseteq \operatorname{Soc}(A f)$ by (2.6) and (3.1), so

$$
\chi^{*} A f=\operatorname{Soc}(A f) \cong M \text {. }
$$

Conversely if $\chi^{*} A f=\operatorname{Soc}(A f)$, then $A \chi^{*} f=\chi^{*} A f \neq 0$ by (2.6), and therefore $\chi^{*} f \neq 0$.

4. Applications. In this section $(A, \varphi)$ is a Frobenius algebra, and $\rho$ is the character of the left regular module ${ }_{A} A$.

LEMma 4.1. Let $\left(a_{i}\right),\left(b_{i}\right)$ be $\varphi$-dual bases for $(A, \varphi)$. Then

$$
\varphi^{-1}(\rho)=\sum_{i} a_{i} b_{i} .
$$


Proof. This proof is identical to that of [3, (3.3)].

Let $M_{1}, \cdots, M_{s}$ be a complete set of (nonisomorphic) simple left $A$-modules with characters $\chi_{1}, \cdots, \chi_{s}$, respectively. Let $m_{i}$ denote the multiplicity of $M_{i}$ as a composition factor of ${ }_{A} A$. It follows that $\rho=\sum_{i=1}^{s} m_{i} \chi_{i}$. If we set $\rho^{*}=\varphi^{-1}(\rho)$ and $\chi_{i}^{*}=\varphi^{-1}\left(\chi_{i}\right)$ for $1 \leqq i \leqq s$, then

$$
\rho^{*}=\sum_{i=1}^{s} m_{i} \chi_{i}^{*}
$$

Now assume $\chi_{k}$ is any irreducible $A$-character, $1 \leqq k \leqq s$. For any $c \in A, c \chi_{k}$ belongs to $M_{k}$, and by (4.2),

$$
\chi_{k}\left(\rho^{*} c\right)=\left(c \chi_{k}\right)\left(\rho^{*}\right)=\sum_{i=1}^{s} m_{i}\left(c \chi_{k}\right)\left(\chi_{i}^{*}\right) .
$$

But by $(3.2),\left(c \chi_{k}\right)\left(\chi_{i}^{*}\right)=0$ unless $M_{i} \cong M_{k}$, i.e., unless $i=k$. Therefore (4.3) implies that $\chi_{k}\left(\rho^{*} c\right)=m_{k}\left(c \chi_{k}\right)\left(\chi_{k}^{*}\right)=m_{k} \chi_{k}\left(\chi_{k}^{*} c\right)$. Together with (2.1) and (4.1) this gives the following (compare [3, (3.12)]).

THEOREM 4.4. Let $\rho$ be the character of the left regular module ${ }_{A} A$, and let $M$ be a simple left $A$-module with character $\chi$. Set $\chi^{*}=$ $\varphi^{-1}(\chi)$. Assume $M$ appears as a composition factor of ${ }_{A} A$ with multiplicity $m$. Then for any $c \in A$,

$$
\chi\left(\rho^{*} c\right)=m \chi\left(\chi^{*} c\right)
$$

In particular if $\left(a_{i}\right),\left(b_{i}\right)$ are $\varphi$-dual bases for $(A, \varphi)$, then for any $c \in A$,

$$
\chi\left(\sum_{i} a_{i} b_{i} c\right)=m \sum_{i} \chi\left(a_{i}\right) \chi\left(b_{i} c\right)
$$

CoRollary 4.5. Let $G$ be a finite group of order $|G|$, and let $K$ be a field of characteristic $p$ such that $p$ does not divide $|G|$. Assume that $K$ is a splitting field for $K G$. Then for any irreducible $K G$-character $\chi$ and for any $h \in G$,

$$
|G| \chi(h)=\chi(1) \sum_{g \in G} \chi(g) \chi\left(g^{-1} h\right) \text {. }
$$

Proof. (Compare [2, (31.6)].) Let $M$ be the simple left $K G$-module with character $\chi$. Since $K G$ is semi-simple (Maschke) and $K$-split, $M$ appears exactly $(M: K)$ times as a composition factor of $K G$, and $\chi(1)=(M: K)$ in $K$. Finally $K G$ is a Frobenius algebra with respect to the particular isomorphism $\varphi$ for which $(g),\left(g^{-1}\right)$ are $\varphi$-dual bases. By (4.4), $|G| \chi(h)=\chi\left(\sum_{g \in G} g g^{-1} h\right)=\chi(1) \sum_{g \in G} \chi(g) \chi\left(g^{-1} h\right)$ as desired.

Assume that $B$ is a simple, finite-dimensional $K$-algebra, $K$ a field, and let $M$ be a simple left $B$-module. Set $D=\operatorname{Hom}_{B}(M, M)$. 
Then $D$ is a division ring, finite-dimensional over $K$, and $B$ is isomorphic to $D_{m}$, the full ring of $m$-by-m matrices over $D$, for some positive integer $m$. It follows that $M$ appears $m$ times as a composition factor of ${ }_{B} B$, and that $(M: K) m=(D: K) m^{2}$. Therefore $(M: K)=$ $(D: K) m$. We combine these remarks with (4.4) to obtain the following.

Corollary 4.6. Assume $(A, \varphi)$ is a Frobenius algebra over a field $K$ of characteristic zero, and let $M$ be a simple projective left $A$-module with character $\chi$. Set $\chi^{*}=\varphi^{-1}(\chi), \rho^{*}=\varphi^{-1}(\rho)$, and $D=$ $\operatorname{Hom}_{A}(M, M)$. Then for some $c \in A, \chi\left(\rho^{*} c\right) \neq 0$, and

$$
(D: K)=\chi\left(\rho^{*} c\right)^{-1} \chi(1) \chi\left(\chi^{*} c\right) \text {. }
$$

In particular if $\left(a_{i}\right),\left(b_{i}\right)$ are $\varphi$-dual bases for $(A, \varphi)$, then for some $c \in A, \chi\left(\sum_{i} a_{i} b_{i} c\right) \neq 0$ and for any such $c$,

$$
(D: K)=\chi\left(\sum_{i} a_{i} b_{i} c\right)^{-1} \chi(1) \sum_{i} \chi\left(a_{i}\right) \chi\left(b_{i} c\right) .
$$

Proof. Because $M$ is projective and $A$ is a $Q F$ ring, the $M$-socle $S_{M}$ of $A$ is a simple block of $A$ to which $M$ belongs by (1.2). Moreover $\chi(1)=(M: K)$ in $K$, and since $K$ has characteristic zero, $\chi \neq 0$. By (3.6), $S_{M}=\chi^{*} A$ and $A=S_{M}+A_{M}$, so $0 \neq \chi(A)=\chi\left(S_{M}\right)=\chi\left(\chi^{*} A\right)$. Therefore for some $c \in A, \chi\left(\chi^{*} c\right) \neq 0$. But (4.4) shows that $\chi\left(\rho^{*} c\right)=$ $m \chi\left(\chi^{*} c\right)$ where $m$ is the multiplicity of $M$ as a composition factor of ${ }_{A} A$, and therefore $\chi\left(\rho^{*} c\right) \neq 0$. The remarks above the statement of this corollary show that $(M: K)=(D: K) m$. Combining these results, we have that

$$
(D: K)=\chi\left(\rho^{*} c\right)^{-1} \chi(1) \chi\left(\chi^{*} c\right) \text {. }
$$

The last statement follows from this, (2.1) and (4.1).

REMARK. The hypothesis that $M$ be projective is essential in the above corollary, for if $M$ is not projective (equivalently, if $\left(\chi^{*}\right)^{2}=0$ ) one can show from (1.2) and (3.6) that $\chi\left(\chi^{*} c\right)=0$ for all $c \in A$.

COROLLARY 4.7. Hypotheses as in (4.6). If $\left(a_{i}\right),\left(b_{i}\right)$ are $\varphi$-dual bases for $A$ such that $\sum_{i} a_{i} b_{i}=(A: K)$, then

$$
(D: K)=(A: K)^{-1} \sum_{i} \chi\left(a_{i}\right) \chi\left(b_{i}\right) \text {. }
$$

Proof. Certainly $\chi\left(\rho^{*}\right)=\chi\left(\sum_{i} a_{i} b_{i}\right)=(A: K) \chi(1) \neq 0$. Therefore we may take $c=1$ in the proof of (4.6), and the conclusion follows. 
REMARKS. One might be surprised to observe that the hypotheses of (4.7) forces $(A, \varphi)$ to be a semi-simple symmetric algebra [3]. For we know that $\rho \in \operatorname{cf}(A)$, so by (2.5) and (4.1), $(A: K)=\sum_{i} a_{i} b_{i}=$ $\varphi^{-1}(\rho) \in Z_{\tau}(A)$. But then $1 \in Z_{\tau}(A)$ and therefore for any $b \in A$, $\mathrm{b}=$ $b \cdot 1=1 \cdot \tau(b)=\tau(b)$. We conclude that $\tau$ is the identity automomorphism which forces $\varphi$ to be an $(A, A)$-bimodule isomorphism (2.3). Therefore $(A, \varphi)$ is a symmetric algebra. Semi-simplicity follows from [3, (1.7)].

If $G$ is a finite group, $K$ a field of characteristic zero which is a splitting field for $K G$ (e.g., $K$ algebraically closed), then the sequence (2.1), (3.1), (3.2), (3.4), (4.1), (4.4) and (4.5) gives an elementary matrix-free proof of the orthogonally relations for irreducible characters of $G$.

The references below include a partial bibliography of papers relating to orthogonality relations for Frobenius algebras.

\section{REFERENCES}

1. R. Brauer, On hypercomplex arithmetic and a theorem of Speiser, Festschrift für Speiser, Zurich, 1945.

2. C. W. Curtis and I. Reiner, Representation Theory of Finite Groups and Associative Algebras, Interscience, New York, 1962.

3. T. V. Fossum, Characters and centers of symmetric algebras, J. of Algebra 16 (1970), 4-13.

4. T. Nakayama, On Frobeniusean algebras I, Math. Ann. 40 (1939), 611-633.

5. - On Frobeniusean algebras II, Math. Ann. 42 (1941) 1-21.

6. Orthogonality relations for Frobenius and quasi-Frobenius-algebras, Proc. Amer. Math. Soc. 3 (1952), 183-195.

7. C. Nesbitt and R. M. Thrall, Some ring theorems with applications to modular representations, Ann. of Math. 47 (1946) 551-567.

8. M. Osima, On the Schur relations for the representations of a Frobenius algebra, J. Math. Soc. of Japan (1952) 1-13.

9. - - Supplementary remarks on the Schur relations for a Frobenius algebra, J. Math. Soc. Japan 5 (1953) 24-28.

Received November 14, 1969.

The University of Utah 
